\title{
First-order sapphire fiber Bragg gratings for high temperature sensing
}

\author{
Tino Elsmann, Tobias Habisreuther, Albrecht Graf, Manfred Rothhardt, Markus A. Schmidt, Hartmut \\ Bartelt, \\ Leibniz-Institut für Photonische Technologien \\ Albert-Einstein-Str. 9, 07745 Jena
}

\begin{abstract}
Summary
Sapphire fiber Bragg gratings (SFBG) are presented as a new kind of high temperature sensor that is not sensitive to electromagnetic fields and can be operated in air as well as inert atmosphere. For inscription of first order gratings femtosecond laser pulses with a wavelength of $400 \mathrm{~nm}$ are applied. Multiplexing of several gratings within one single crystal fiber is demonstrated. The gratings were tested for short term up to $1,900^{\circ} \mathrm{C}$ and for a period of 28 days at $1,400^{\circ} \mathrm{C}$. An adapted signal processing of the reflexion spectra of the highly multimode air clad sapphire fibers with a diameter of $100 \mu \mathrm{m}$ allows detecting relative temperature changes better than $\pm 1 \mathrm{~K}$. Tubes can protect the air clad sapphire fiber from reactions with the environment. Applying a bare fiber allows to detect temperature changes with several $\mathrm{Hz}$ speed. Strain sensor designs and strain measurements based on SFBG for applications up to $600^{\circ} \mathrm{C}$ were tested, too. The sensitivity of multimode SFBG strain sensors is $\Delta \mathrm{l} / \mathrm{I}=$

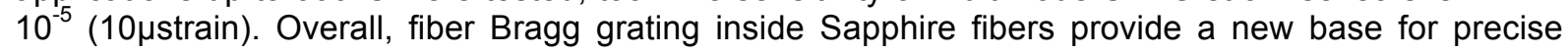
high-temperature sensors with key advantages such as signal multiplexing, large temperature bandwidth and insensitivity to electromagnetic fields.
\end{abstract}

Keywords: high-temperature sensor, strain sensor, fiber optic sensor, sapphire fiber

\section{introduction}

High temperature processes are of great importance in production and optimization of many industrially-relevant processes. Temperatures higher than $1000^{\circ} \mathrm{C}$ are applied for melting of metals or glass, crystal growth or the fabrication of ceramics. For a more efficient and safe energy supply high-temperature processes in heat engines or in reactor cores need to be optimized.

Various sensor concepts for measuring such high temperatures precisely are available, but the optimisation of high temperature sensors is an ongoing research activity [1]. Noble metal thermocouples can be used in air up to $1,700^{\circ} \mathrm{C}$. Thermocouples therefore require regular checks or might even need to be replaced due to drifts caused by diffusion processes [2] In an electro-magnetically polluted environment metal based sensors may not be applicable.

Pyrometers respond fast, allow studying dynamic, and can be operated in different atmospheres at temperatures $>2000^{\circ} \mathrm{C}$ [3-7]. But they require direct optical access to the hot surface. Changes in the emissivity of the hot surface may lead to errors in the measurements.
Fiber optic based sensors are an alternative [8]. They operate in various atmospheres and are not influenced by electromagnetic fields. Conventional optical fiber sensors are based on fused silica. The glass transition temperature at about $1200^{\circ} \mathrm{C}$ limits the application of these sensors. One promising candidate measuring at higher temperatures are single crystalline sapphire fibers. They can be used up to their melting point of about $2050^{\circ} \mathrm{C}$. Using femtosecond laser sources fiber Bragg gratings can be inscribed in the single crystalline sapphire fiber (SFBG) [9]. Using the phase-mask technology at a wavelength of $800 \mathrm{~nm}$ was leading to second order gratings. They were tested up to temperatures of $1740^{\circ} \mathrm{C}$ [10]. Using a Talbot interferometer and an inscription wavelength of $400 \mathrm{~nm}$ allows the inscription of first order gratings and grating arrays [11].

In this paper the temperature dependence of SFBGs and their application as temperature sensors at very high temperatures between $1,200^{\circ} \mathrm{C}$ and $1,900^{\circ} \mathrm{C}$ as well as for fast responding sensors will be presented. As a special application the temperature distribution in an inductively heated tube furnace will be discussed. 


\section{Inscription of sapphire fiber Bragg gratings}

A Ti:sapphire-amplified femtosecond laser system was used for the inscription of FBGs. Pulses with a wavelength of $800 \mathrm{~nm}$, a pulse duration of 135 fs and an averaged power of 3 W with a repetition rate of $1 \mathrm{kHz}$ pass a nonlinear crystal to generate the second harmonic of the pump wave leading to a wavelength of $400 \mathrm{~nm}$ with an averaged power of $1 \mathrm{~W}$. The high peak intensities of these pulses and multi-photon processes lead to a permanent change of the refractive index and thus to the inscription of FBGs.

To avoid a material ablation an external dynamic iris diaphragm for a reduction of the mean repetition rate and a reduction of the laser power to about $550 \mathrm{~mW}$ were applied. With the iris diaphragm an average of 20 pulses per second reached the fiber.

The FBGs were fabricated using the interference pattern of a Talbot interferometer (see Fig. 1) [11,12]. The beam is divided by a phase mask with a period of $888 \mathrm{~nm}$, optimized for an inscription wavelength of $\lambda_{\text {inscription }}=400$ $\mathrm{nm}$. The two diffracted beams were reflected by mirrors and then interfered under the angle $\theta$. The fiber has to be placed exactly perpendicular to the interference pattern in the field of superposition. An additional cylindrical lens with a focal length of $221 \mathrm{~mm}$ was used in front of the interferometer to increase the local intensity at the place of the fiber. Since the diameter of sapphire fibers was $100 \mu \mathrm{m}$ and the focal width was about $10 \mu \mathrm{m}$ the cylindrical lens was moved to scan through the full fiber diameter with a speed of $0.1 \mu \mathrm{m} / \mathrm{s}$. The tolerance for the alignment of the two beam paths in the interferometer is less than 50 microns due to the pulses in the fs- regime. Turning the mirrors symmetrically leads to a change in the angle $\theta$ leading to a change in

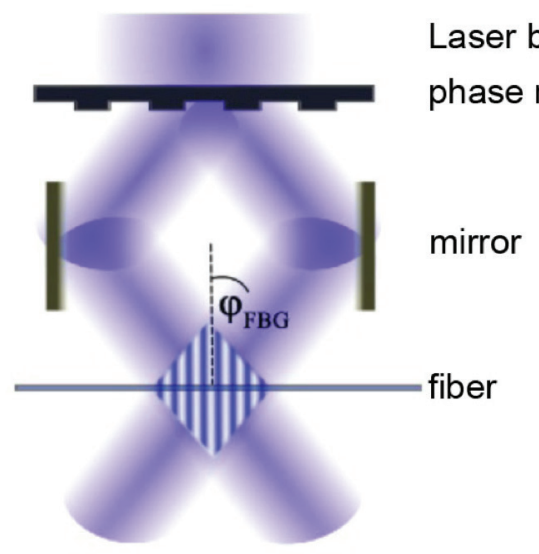

Fig. 1: Talbot interferometer used for FBG inscription

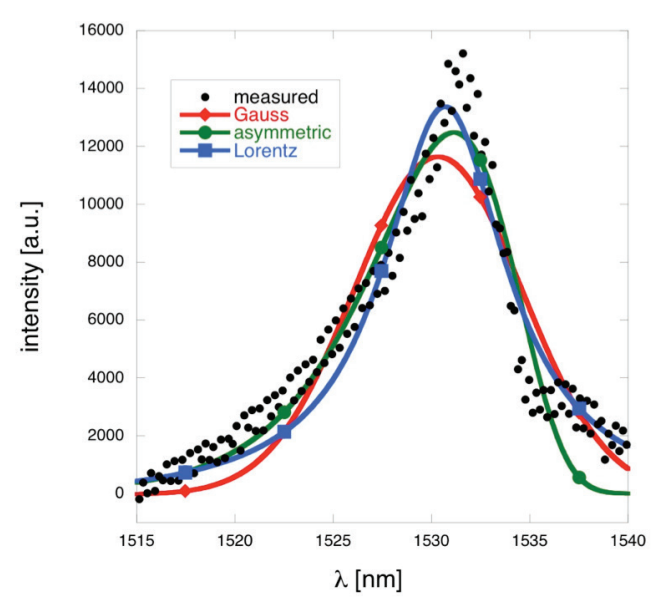

Fig. 2: measured peak-spectrum for sapphire FBG (black dots) and different fittted peak functions

the design Bragg wavelength (Eq. 1) and enables multiplexing of gratings, where $n$ is the refractive index of the fiber.

$\lambda_{\text {Bragg }}=\left(n \lambda_{\text {nscription }}\right) /(\sin \theta) \quad(E q .1)$,

We used commercial single crystalline sapphire fibers (MicroMaterials Inc.) with lengths up to one meter fabricated by laserheated pedestal growth. Sapphire fibers are core only air clad multimode core fibers with an index difference of 0.74 relative to air. Several thousand modes can propagate through the fiber, which results in a very broad reflection spectrum of the FBG of about more than $8 \mathrm{~nm}$ (fig. 2).

The interrogation setup is shown in Fig. 3. For illumination we use a SLD light source with a Gaussian-like intensity distribution in the spectral range from 1510 to $1590 \mathrm{~nm}$, a commercial FBG interrogator (Ibsen Photonics) modified by a $50 \mu \mathrm{m}$ graded-index (GI) fiber entrance, a $50 \mu \mathrm{m}$ GI fiber-optic $Y$-coupler, and a $50 \mu \mathrm{m}$ Gl launch fiber with a length of $100 \mathrm{~m}$ as mode scrambler to excite all propagating modes homogeneously. The sapphire fiber and the launch fiber are coupled via a commercial APC connector. We observe some losses

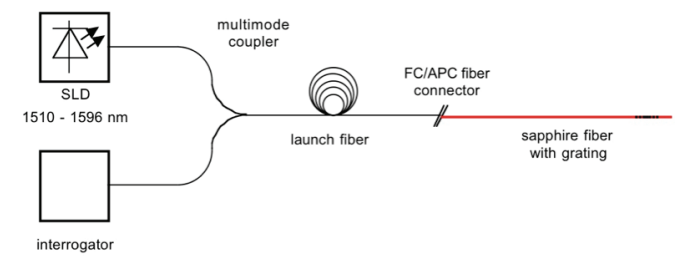

Fig. 3: interrogation scheme for sapphire FBG 
especially for the coupling from $100 \mu \mathrm{m}$ sapphire to the $50 \mu \mathrm{m}$ supply fiber, but the signal output is well measurable due to a grating reflectivity of about $10 \%$.

\section{Signal processing}

To obtain a fiber Bragg wavelength from this spectrum, one possibility is to extract the lowest order mode by tapered single mode fiber [13]. Our approach was to use the whole spectrum gaining better sensitivity, and maintaining sensor robustness and costefficient fabrication as well. Several peak functions can be fitted to the whole spectrum (Fig. 2): a Gauss-distribution (eq. 2), a Lorentz function (eq. 3), and an asymmetric peak function (eq. 4). There is no physical law that justifies any of the chosen functions. Especially the asymmetric function was selected because of the better shape fitting.

$$
\begin{aligned}
& y=y_{0}+\frac{A}{w \sqrt{\pi / 2}} e^{-2 \frac{\left(x-x_{c}\right)^{2}}{w^{2}}} \\
& y=y_{0}+\frac{2 A}{\pi} \frac{w}{4\left(x-x_{c}\right)^{2}+w^{2}} \\
& y=y 0+A e^{\left(-e^{(-z)}-z+1\right)} \\
& z=(x-x c) / w
\end{aligned}
$$

The Bragg wavelength $\lambda_{B}\left(x_{C}\right.$ in the formula) differs for each of these functions. But according to our investigation the temperature sensitivity is independent of the chosen peak function. The asymmetric function was applied for the further investigations.

As an example the fit results in a Bragg wavelength $\lambda_{B}=1543.964 \pm 0.119 \mathrm{~nm}$ at $600^{\circ} \mathrm{C}$. With a sensor sensitivity of $23 \mathrm{pm} / \mathrm{K}$ fitting a single spectrum results in a measurement accuracy of $\pm 6 \mathrm{~K}$. The peak width is $7.0 \mathrm{~nm}$. For an improvement of the temperature resolution a floating mean procedure was checked. Figure 6 shows the mean Bragg wavelength in dependence on the number of averaged fitted Bragg wavelengths.

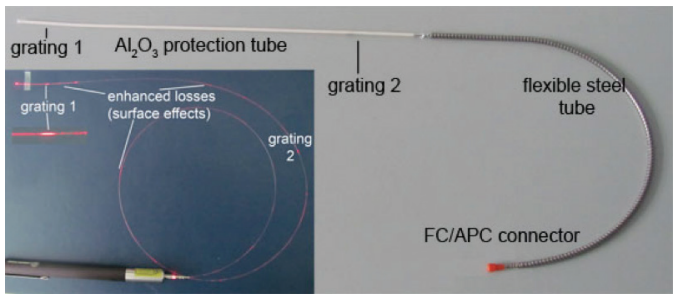

Fig. 4: example for a sapphire FBG temperature sensor (inset: sapphire fiber illuminated by red light)
The error bars indicate the statistic error. For an averaging of 20 fitted Bragg wavelengths the mean Bragg wavelength is constant with a statistic variance of below $10 \mathrm{pm}$. In this way, temperatures change can be determined with a resolution of about $1 \mathrm{~K}$ [14].

\section{Sensor packaging}

To provide a signal that is not influenced by the environment the sapphire fiber has to be protected. In conventional fused silica based optical fibers light is guided in the fiber core. Cladding and coating protect the guided light from the environment. Sapphire fibers are air clad. At present neither cladding nor coating are available that do not react with sapphire at elevated temperatures $>1200^{\circ} \mathrm{C}$. Therefore we place the sapphire fibers in closed tubes from sintered $\mathrm{Al}_{2} \mathrm{O}_{3}$ or even single crystal sapphire. We did not observe disturbances to the signal from the mechanical contact between sapphire fiber and tube wall. The fiber packaging leads to temperature probes quite similar to thermocouples that are protected by ceramic tubes as well. The ceramic protection can be combined with a flexible metal hose that protects the sapphire fiber at moderate temperatures. An example of this design is shown in fig. 4.

\section{Temperature dependence}

The temperature dependence of the SFBG wavelength is shown in Fig. 5. The large red squares for $\mathrm{T}<1,200^{\circ} \mathrm{C}$ represent data after the temperature in the calibration furnace (METEK CTC-1200A) was stabilized for more than $7 \mathrm{~min}$. The green solid line represents data during the slow heating with a rate of $3 \mathrm{~K} / \mathrm{min}$ of the furnace match these data as well. The

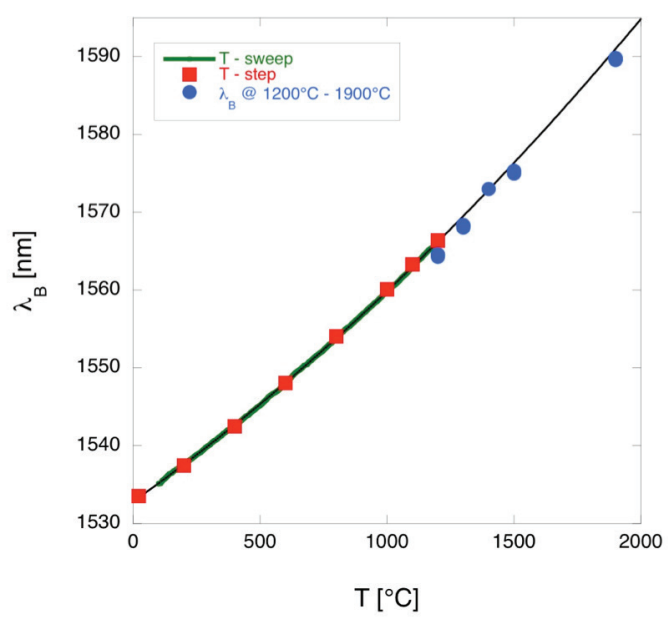

Fig. 5: temperature calibration of sapphire FBG 
dependency of $\lambda_{B}$ on temperature is nonlinear. A second order polynomial was fitted to the data up to $1,200^{\circ} \mathrm{C}[15]$ :

$$
\begin{aligned}
\lambda_{\mathrm{B}}(\mathrm{T})=1,532.8 \mathrm{~nm}+0.023 \mathrm{~nm} /{ }^{\circ} \mathrm{C} \cdot \mathrm{T} \\
+4 \cdot 10^{-6}\left(\mathrm{~nm} /{ }^{\circ} \mathrm{C}^{2}\right) \cdot \mathrm{T}^{2} \quad(\text { eq. } 5)
\end{aligned}
$$

With increasing temperature the sensitivity of the SFBG sensors grows from $23 \mathrm{pm} / \mathrm{K}$ at room temperature to $35 \mathrm{pm} / \mathrm{K}$ at $1500^{\circ} \mathrm{C}$.

An extrapolation of the fitting curve up to $2000^{\circ} \mathrm{C}$ was performed. (black line in Fig. 4). Bragg wavelengths measured in an inductive furnace are plotted by blue dots. The data follow the extrapolated fitting curve up to $1,900^{\circ} \mathrm{C}$. Deviations from the curve may be caused by thermal fluctuations in the inductively heated furnace induced by the flowing Ar-gas.

\section{Temperature distribution of an inductively heated furnace}

As an example for application we determined the temperature distribution within the hot zone of an inductively heated furnace at $1200^{\circ} \mathrm{C}$ and $1500^{\circ} \mathrm{C}$. The heated tube zone has a length of about $4 \mathrm{~cm}$ and a diameter of $4.5 \mathrm{~cm}$. The sensor was fixed at different radial positions and slowly scanned parallel to the tube axis. The figures $6 a$ and $6 b$ represent the temperature in a half-sphere from the center to
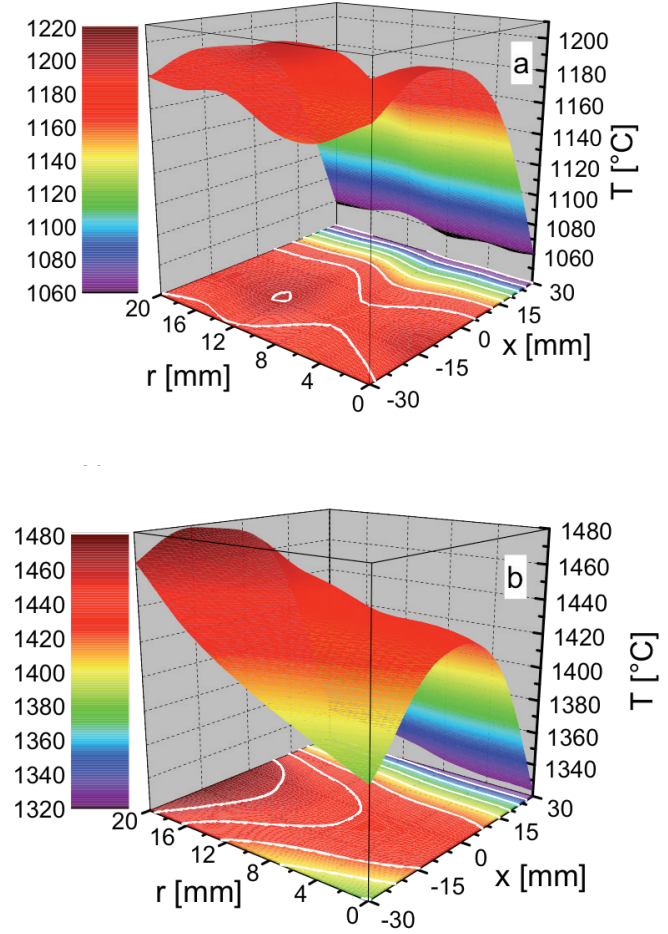

Fig. 6: temperature distribution (half plane) in a inductively heated tube furnace at a) $1200^{\circ} \mathrm{C}$ and b) $1500^{\circ} \mathrm{C}$ the furnace wall and along the furnace axis. At $1200^{\circ} \mathrm{C}$ there is no significant temperature variation from the center axis to the furnace wall. Also the homogeneous zone along the furnace axis has a length of about $2.5 \mathrm{~cm}$. At $1500^{\circ} \mathrm{C}$ the temperature distribution is less homogeneous. From the center to the wall there is a temperature increase of about $40 \mathrm{~K}$. Also the temperature distribution along the furnace axis is less homogeneous. This effect may be explained by flowing Argon during the furnace operation. The Ar-gas is not heated up, therefore it may act cooling. Furthermore the furnace was operated with the tube opened. Heat radiation to the environment leads to the spatial reduction of the homogeneous heated zone.

\section{Long term stability}

For investigations of the long term stability, a sensor arrays consisting of two different SFBGs was prepared. The first grating was placed near to the end of the fiber and its Bragg wavelength was at about $1545 \mathrm{~nm}$ at room temperature. The second grating was inscribed at a distance of $25 \mathrm{~cm}$ to the first grating with a Bragg wavelength of about 1525 $\mathrm{nm}$ at room temperature. The first grating SFBG1 was exposed to high temperatures in a furnace whereas the second grating SFBG2 was placed outside but close to the furnace at moderate temperatures up to about $150^{\circ} \mathrm{C}$. Again, for protection and better handling, the Sapphire fiber was placed in an $\mathrm{Al}_{2} \mathrm{O}_{3}$ ceramic tube.

After installation of the Sapphire sensors together with a type B thermocouple at room temperature, the furnace was heated up to $1400^{\circ} \mathrm{C}$ within one day. Then, this temperature

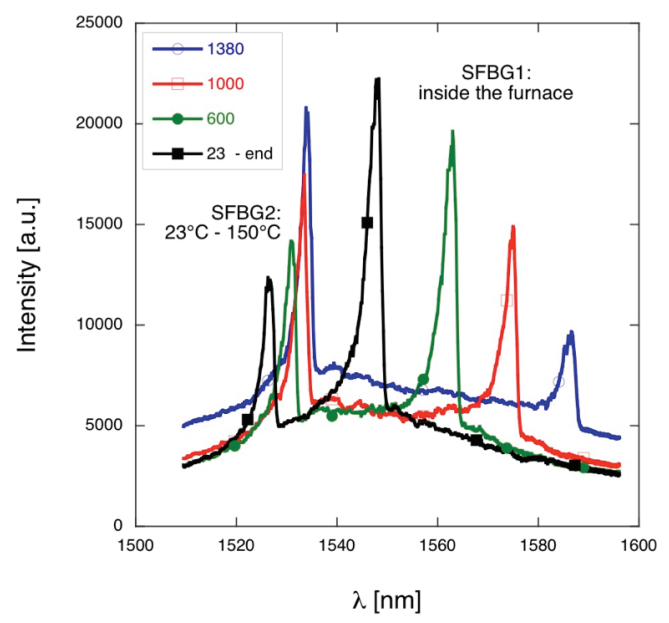

Fig. 7: temperature dependent spectra of an array exposed up to $1400^{\circ} \mathrm{C}$. 


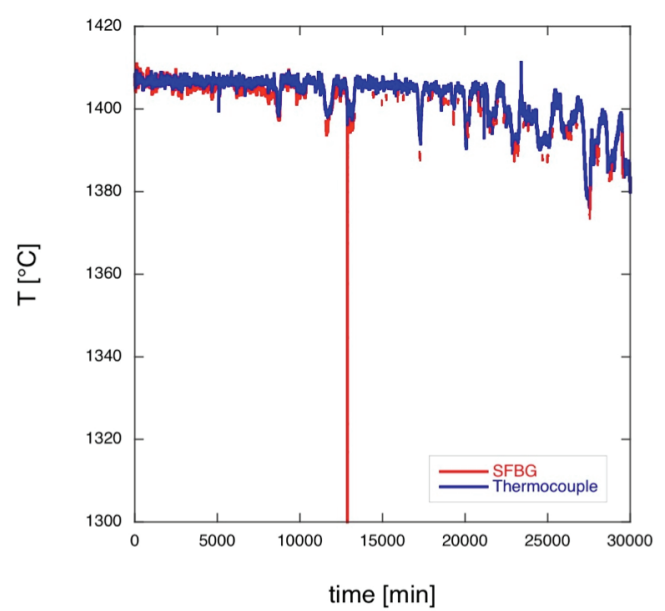

Fig. 8: comparison between sapphire FBG and thermocouple. No significant diffenences were observed over 4 weeks

inside the furnace was tried to keep stable for 28 days. In the first week of the test period the temperature was nearly stable to about $5^{\circ} \mathrm{C}$, then temperature oscillations in the furnace were launched.

Figure 7 shows the change in the spectra at different temperatures of the furnace. As it can be seen from the graph the peak position of grating 2 out of the furnace shows a slight drift caused by the temperature increase from room temperature to $150^{\circ} \mathrm{C}$. The Bragg wavelength of grating 1 inside the furnace shifts from 1547 $\mathrm{nm}$ at room temperature to $1587 \mathrm{~nm}$ at $1400^{\circ} \mathrm{C}$. The peak intensity change of grating 1 is caused by the spectral intensity distribution of the SLD.

In Fig. 8 it is shown that temperatures measured by both types of sensors are very similar. During the first period both sensor types show stable temperatures, then both types show identical oscillations. For the whole period of the installation the differences between the two thermal sensors are less than $2 \mathrm{~K}$.

The sharp drop down at about 13.000 min was caused by insertion of a second thermocouple for control into the furnace. As the interrogation rates of $\Delta t=10 \mathrm{~s}$ for the sapphire $F B G$ and $\Delta t$ $=10 \mathrm{~min}$ at that moment differed the effect was visualized by the sapphire FBG. Comparing the spectra at the beginning and the end of the installation did not show a difference. The sapphire temperature sensor was stable.

\section{Strain sensing}

For a fiber Bragg grating strain $\varepsilon$ leads to a shift $\Delta \lambda$ in the Bragg wavelength at constant temperature. ( $\lambda_{B}$ is the Bragg wavelength, $p$ the thermo-mechanic coefficient)

$$
\varepsilon=\Delta \lambda /\left(\lambda_{B} \cdot(1-p)\right) \quad(E q .3)
$$

In comparison to fused silica fibers where $p \approx 0.22$ [16] the value for sapphire is $p=0.13$ [17], leading to a enhanced sensitivity by about $1.4 \mathrm{pm} / \mu$ strain.

The cross sensitivity between temperature and strain of sapphire fibers has to be considered. A change in temperature of $1 \mathrm{~K}$ corresponds to an applied strain of about $20 \mu$ strain. The Bragg wavelength can be detected with a resolution of about $10 \mathrm{pm}$, so the sensitivity of sapphire FBG is $10 \mu$ strain. This requires that temperatures are determined with an accuracy of $0.5 \mathrm{~K}$.

A strain sensor requires a force fit connection between the sapphire fiber and the device under investigation. We tested a simple design: A base steel plate $\left(20 * 50 * 1 \mathrm{~mm}^{3}\right)$ with a $V$-groove acts as sensor pad which can be spot-welded to the device under investigation. The SFBG is in-between the two gluing spots that fix the fiber in the $V$-groove. A tube which is half cut on a length of about $4 \mathrm{~cm}$ is fixed over the sapphire fiber for mechanical protection.

The temperature characteristics of the strain sensor is shown in Fig. 9. The metal substrate used for second sensor design was prepared from a special high temperature tough steel alloy containing $9 \%$ chromium applied in coal power plant steam pipes.

The figure shows the temperature dependence of the SFBG before it was applied to the sensor pad (red curve). Also the wavelengths during the glassing of the adhesive the blue

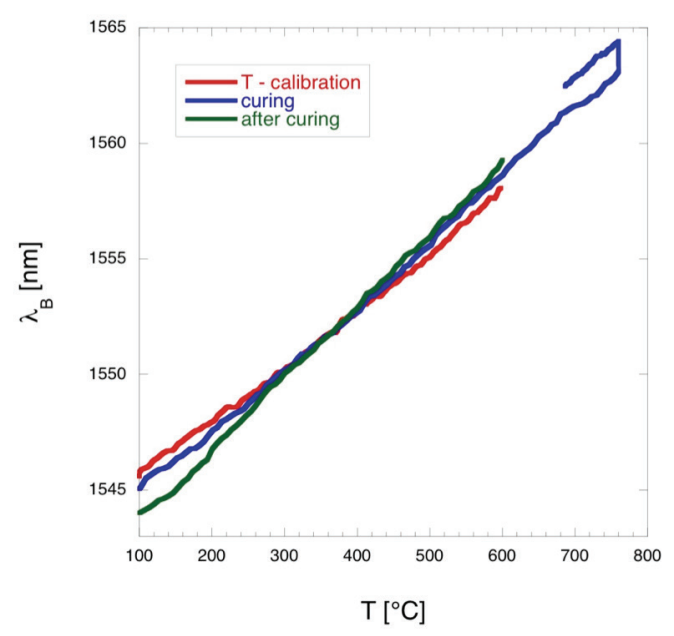

Fig. 9 sapphire strain sensor. The different slope of the heating curves indicates that the sapphire fiber was strained. 
curve was measured. For $\mathrm{T}>700^{\circ} \mathrm{C}$ glassing of the adhesive begins, which leads to the desired force fit contact between the steel pad and the sapphire fiber. During the subsequent heating cycle after curing (green curve) the temperature dependence differs from the temperature calibration before. As the adhesive reacted at elevated temperatures, the fiber in fact is compressed for temperatures below $350^{\circ} \mathrm{C}$, which is indicated by the fact that the measured Bragg wavelength at $100^{\circ} \mathrm{C}$ of the fixed sensor is, at the same temperature, below the calibration Bragg wavelength.

From Fig. 7 the linear temperature dependence in the range $100-600^{\circ} \mathrm{C}$ for the fiber was determined to $24.3 \mathrm{pm} / \mathrm{K}$, whereas the fixed sensor shows a slope of $30.1 \mathrm{pm} / \mathrm{K}$.

From the difference of $5.8 \mathrm{pm} / \mathrm{K}$ the difference in thermal expansion between sapphire and the steel calculates to $4.3 \cdot 10^{-6} / \mathrm{K}$ resulting in an expansion coefficient for the metal of $11.5 \cdot 10^{-6} / \mathrm{K}$. Measuring the thermal expansion coefficient by dilatometer confirmed this value.

\section{Summary}

We presented fiber Bragg gratings inscribed in single crystal sapphire fibers as the base of new high temperature sensors. SFBGs can be multiplexed and enable temperature measurement in any atmosphere from room temperature up to $1900^{\circ} \mathrm{C}$. Temperature resolution of $1 \mathrm{~K}$ is possible. SFBGs also can be applied for strain sensing. Here a sensitivity of $10 \mu$ strain can be reached.

\section{Acknowledgment}

This work was supported by the German Federal Ministry of Economics and Technology under contract 13INE036, and the Thuringian Ministry of Education, Science and Culture (EFRE program).

\section{References}

[1] G. Machin, "HiTeMS: A pan-European project to solve high temperature measurement problems in industry", MEASUREMENT \& CONTROL 45 (10), 315-318 (2012)

[2] S. Jun, O. Jochan, "Investigations on Thermocouple drift irregularity impact on their inhomogeneity correction", Measurement science review, 14 (1), 29 (2014)

[3] G.M. Foley, "High Speed Optical Pyrometer", Review of Scientific Instruments 41, 827 (1970)

[4] M. B. Boslough, T. J. Ahrens, "A sensitive time resolved radiation pyrometer for shocktemperature measurements above 1500 K", Review of Scientific Instruments 60, 3711 (1989)

[5] J. Thevenet, M. Siroux, B. Desmet, "Measurements of brake disc surface temperature and emissivity,by two-color pyrometry", Applied Thermal Engineering 30, $753(2010)$

[6] J. Bezemer, "Spectral Sensitivity Corrections for Optical Standard Pyrometers", Metrologia 10, 47-52 (1974)

[7] B. Müller, U. Renz, "Development of a fast fiberoptic two-color pyrometer for the temperature measurement of surfaces with varying emissivities", Review of Scientific Instruments 72, 3366 (2001)

[8] M. Willsch, T. Bosselmann, P. Flohr, R. Kull, W. Ecke, I. Latka, D. Fischer, "Design of Fiber Optical High Temperature Sensors for Gas Turbine Monitoring", Proc. of SPIE Vol. 7503, 75037 (2009)

[9] D. Grobnic, S. J. Mihailov, C. W. Smelser, H. Ding, "Sapphire fiber Bragg grating sensor made using femtosecond laser radiation for ultrahigh temperature applications", IEEE PHOTONICS TECHNOLOGY LETTERS 16 (11), 2505-2507 (2004)

[10] M. Busch, W. Ecke, I. Latka, D. Fischer, R. Willsch, H. Bartelt, "Inscription and characterization of Bragg gratings in singlecrystal sapphire optical fibres for hightemperature sensor applications", Meas. Sci. Technol. 20(11), 115301 (2009)

[11] T. Elsmann, T. Habisreuther, A. Graf, M. Rothhardt, H. Bartelt, "Inscription of first-order sapphire Bragg gratings using $400 \mathrm{~nm}$ femtosecond laser radiation", OPTICS EXPRESS 21 (4), 4591 (2013)

[12] M. Becker, J. Bergmann, S. Brückner, M. Franke, E. Lindner, M. W. Rothhardt, H. Bartelt, "Fiber Bragg grating inscription combining DUV sub-picosecond laser pulses and two-beam interferometry," Opt. Express 16 (23), 1916919178 (2008).

[13] D. Grobnic , S. Mihailov, H. Ding, F. Bilodeau, C. W. Smelser, "Single and low order mode interrogation of a multimode sapphire fibre Bragg grating sensor with tapered fibres.", Measur. Sci. Technol. 17, 980-984 (2006).

[14] T. Habisreuther, T. Elsmann, Z. Pan, A. Graf, H.-J. Pißler, M. Rothhardt, R. Willsch, H. Bartelt, M.A. Schmidt, "Optical sapphire fiber Bragg gratings as high temperature sensors", Proc. SPIE 8794, 87940B (2013)

[15] T. Habisreuther, T. Elsmann, Z. Pan, A. Graf, R. Willsch, M. A. Schmidt, "Sapphire fiber Bragg gratings for high temperature and dynamic temperature diagnostics," Applied Thermal Engineering, vol. 91, 860-865 (2015)

[16] D. Kersey, M. A. Davis, H. J. Patrick, M. LeBlanc, K. P. Koo, C. G. Askins, M. A. Putnam, E. J. Friebele, "Fiber Grating Sensors", JOURNAL OF LIGHTWAVE TECHNOLOGY 15 (8), 1442 (1997)

[17] S. Mihailov, D. Grobnic, C. Smelser, "Hightemperature multiparameter sensor based on sapphire fiber Bragg gratings," OPTICS LETTERS, vol. 35 no. 16, 2810-2812, (2010) 